\title{
Relationship Between Salivary Pepsin Concentration and Esophageal Mucosal Integrity in Patients With Gastroesophageal Reflux Disease
}

\author{
Yu-wen Li, ${ }^{1}$ Daniel Sifrim, ${ }^{2}$ Chenxi Xie, ${ }^{1}$ Minhu Chen, ${ }^{1 *}$ and Ying-lian Xiao ${ }^{1 *}$ \\ ${ }^{I}$ Department of Gastroenterology, First affiliated Hospital, Sun Yat-sen University, Guangzhou, GuangDong Province, China; and \\ ${ }^{2}$ Wingate Institute of Neurogastroenterology, Queen Mary University of London, London, UK
}

\section{Background/Aims}

Increased salivary pepsin could indicate an increase in gastro-esophageal reflux, however, previous studies failed to demonstrate a correlation between salivary pepsin concentrations and 24-hour esophageal acid exposure. This study aims to detect the salivary pepsin and to evaluate the relationship between salivary pepsin concentrations and intercellular spaces (IS) in different gastroesophageal reflux disease phenotypes in patients.

\section{Methods}

A total of 45 patients and 11 healthy volunteers were included in this study. All subjects underwent upper gastrointestinal endoscopy, 24-hour ambulatory multichannel impedance-pH (MII-pH) monitoring, and salivary sampling at 3-time points during the 24-hour MII-pH monitoring. IS were measured by transmission electron microscopy, and salivary pepsin concentrations were determined by enzyme-linked immunosorbent assay.

\section{Results}

The IS measurements were greater in the esophagitis (EE), non-erosive reflux disease (NERD), and hypersensitive esophagus (HO) groups than in the functional heartburn (FH) and healthy volunteer groups, and significant differences were indicated. Patients with NERD and HO had higher average pepsin concentrations compared with FH patients. A weak correlation was determined between IS and salivary pepsin among patients with NERD $(r=0.669, P=0.035)$.

\section{Conclusions}

We confirmed the presence of a higher level of salivary pepsin in patients with NERD than in patients with FH. Salivary pepsin concentrations correlated with severity of mucosal integrity impairment in the NERD group. We suggest that in patients with NERD, low levels of salivary pepsin can help identify patients with $\mathrm{FH}$, in addition the higher the pepsin concentration, the more likely the severity of dilated IS.

(J Neurogastroenterol Motil 2017;23:517-525)

\section{Key Words}

Acid exposure time; Dilated intercellular spaces; Gastroesophageal reflux disease; Salivary pepsin

Received: October 13, 2016 Revised: May 1, 2017 Accepted: Aug 16, 2017

(.) This is an Open Access article distributed under the terms of the Creative Commons Attribution Non-Commercial License (http://creativecommons. org/licenses/by-nc/4.0) which permits unrestricted non-commercial use, distribution, and reproduction in any medium, provided the original work is properly cited.

${ }^{*}$ Correspondence: Minhu Chen and Ying-lian Xiao are equally responsible for this study. Minhu Chen, MD Department of Gastroenterology and Hepatology, First affiliated hospital, Sun Yat-sen University, Guangzhou 510080, China Tel: +86-020-28823388-8155, Fax: +86-02087333122, E-mail: chenminhu@vip.163.com Ying-lian Xiao, MD Department of Gastroenterology and Hepatology, First affiliated hospital, Sun Yat-sen University, Guangzhou 510080, China Tel: +86-020-28823388-8455, Fax: +86-02087333122, E-mail: yinglian_xiao@163.com 


\section{Introduction}

Gastroesophageal reflux disease (GERD) is a common disorder which develops when reflux of gastric contents causes uncomfortable symptoms and/or complications. ${ }^{1}$ The prevalence of GERD has increased both in Western countries and Asia. ${ }^{2}$ Diagnostic methods for GERD include GERD-specific questionnaires, gastrointestinal (GI) endoscopy, ambulatory reflux monitoring, and treatment with empirical proton pump inhibitors (PPIs). Unfortunately, these approaches fail to achieve high sensitivity and specificity, particularly in patients with non-erosive reflux disease (NERD). In addition, some of these methods may be poorly tolerated, invasive, and costly. Endoscopy can detect erosive esophagitis (EE). However, most patients with heartburn and regurgitation have no erosive disease. ${ }^{3}$ Reflux monitoring with $\mathrm{pH}$ or impedance$\mathrm{pH}$ metry is also considered critical for the diagnosis of GERD. However, this measurement enables only short-term analysis ${ }^{4}$ and includes certain disadvantages, such as patient discomfort and catheter displacement rendering the test cumbersome and error-prone. ${ }^{5}$ Moreover, patients cannot tolerate the catheter-based test. ${ }^{6}$ Dietary modifications can lead to false negative study results. Empirical PPI treatment exhibits moderate sensitivity and low specificity for the diagnosis of GERD. ${ }^{7}$ Several studies have demonstrated various ultrastructural lesions in patients with GERD, with dilated intercellular spaces (DIS) being one of the common changes. ${ }^{8}$ DIS occurs mainly in the basal layer of patients with GERD. DIS can favor luminal ion flow through the epithelium, which could activate epithelial and neural receptors leading to symptoms. ${ }^{9,10}$ Some researchers have suggested that DIS can be used as an objective indicator for NERD. ${ }^{11,12}$ However, this method of GERD diagnosis is invasive and costly. Therefore, researchers have shifted their attention toward a simpler and less expensive technique: saliva pepsin testing.

Pepsin plays an important role in the pathophysiology of GERD. ${ }^{13} \mathrm{~A}$ recently published article has found that in patients with symptoms suggestive of GERD, testing for salivary pepsin may facilitate the diagnosis of GERD, the study also suggested that salivary pepsin may reduce the use of unnecessary antireflux therapy and the need for further invasive and expensive diagnostic methods. ${ }^{14}$ Saliva can easily be obtained in many patient groups (particularly in adults, children, and neurologically impaired patients) and can be easily detected. ${ }^{15}$ However, high variation in salivary pepsin is present, increased salivary pepsin could indicate an increase in acid reflux, although a good correlation between salivary pepsin concentrations and 24-hour esophageal acid expo- sure was not demonstrated in previous studies. Another possibility could be that salivary pepsin concentration correlates better with a more stable or chronic manifestation of reflux, such as microscopic mucosal damage, rather than a variable 24-hour reflux measurement. The relationship between salivary pepsin concentration and esophageal mucosal integrity measured in intercellular spaces (IS) remains undetermined. This study aims to evaluate the relationship between salivary pepsin concentrations and IS in different GERD phenotypes in patients.

\section{Materials and Methods}

\section{Study Design}

Studies were performed on 45 patients who presented with recurrent heartburn and/or regurgitation ( $\geq 2$ days/week). The symptoms had lasted for at least 3 months. All patients manifested at least moderate reflux symptoms. Patients were excluded if any one of the following conditions was present: (1) peptic ulcer, erosive gastritis, or GI tumors detected by endoscopy; (2) inability to complete all investigations, (3) women in pregnancy or lactation; and (4) severe systemic diseases (such as severe heart, liver, kidney, brain, and other organ damage).

All patients underwent upper GI endoscopy and 24-hour ambulatory multichannel impedance-pH (MII-pH) monitoring after at least 1 week of pharmacological washout.

Eleven healthy volunteers without any GI symptoms during the last 3 months were also recruited for comparison. Signed informed consent was obtained from each individual prior to study inclusion. The study was approved by the Ethics Committee of the First Affiliated Hospital of Sun Yat-Sen University.

\section{Upper Gastrointestinal Endoscopy and Preparation of Esophageal Biopsies for Electron Microscopy}

All patients underwent upper GI endoscopy after overnight fasting. Esophageal biopsy was performed by taking a tissue 2-4 $\mathrm{cm}$ above the gastroesophageal junction Z-line, and immediately placing into $2.5 \%$ glutaraldehyde- $2 \%$ paraformaldehyde- $0.1 \mathrm{~mol} /$ $\mathrm{L}$ phosphate buffer for 4 hours. The tissue was washed using a buffer solution 3 times, with each wash-out period lasting for 10 minutes, and then fixing in $1 \%$ osmic acid- $0.1 \mathrm{~mol} / \mathrm{L}$ phosphate buffer for 1.5 hours. Three additional wash-outs were performed using the same method. The tissue was then dehydrated with $50 \%$, $70 \%, 80 \%, 90 \%$, and $100 \%$ of ethanol, minimum exposure to each medium for 10-15 minutes, followed by exposure to $100 \%$ acetone 
twice, each time lasting for 10-15 minutes. The tissue was placed in acetone:embedding medium $=1: 1$, soaked for 1 hour, and then acetone:embedding medium $=1: 2$, soaked for 3 hours. The tissue was placed in a pure embedding medium for overnight soaking, picked and placed into the embedding plate for aggregation at $60^{\circ} \mathrm{C}$ for about 48 hours to harden. The tissue was sliced into $1-2$ $\mu \mathrm{m}$ sections to locate the target and then into 50-60 nm ultrathin sections and pulled into the copper grid. Saturated uranium acetate and lead citrate were used to dye the tissue.

\section{Electron Microscopy}

Distal esophageal biopsies were examined using an electron microscope (Tecnai G2 Spirit Twin; FEI company, Hillsboro, Oregon, USA) at $\times 5000$ magnification. For each biopsy, 10 randomly selected fields from the basal cell of the epithelial layer were photographed for subsequent analysis. Image-Pro Plus 6.0 software (Media Cybernetics, Inc, Rockville, MD, USA) was used to measure the IS. Mean IS in $\mu \mathrm{m}$ was calculated in each subject by using the 10 photographs, and in each photograph the average diameters in 10 random transects (drawn perpendicular to the neighboring membranes) was evaluated, and no 2 transects were drawn closer than $1 \mu \mathrm{m}$ apart. This procedure resulted in 100 transects available for IS measurement in each patient, as previously described by Tobey et al. ${ }^{16}$ IS measurement was conducted in a blinded manner by 2 observers, with no knowledge of the study groups of subjects.

\section{4-Hour Ambulatory Multichannel Impedance-pH Monitoring}

The MII-pH monitor assembly (Sandhill Scientific Inc, Highlands Ranch, CO, USA) was positioned with the proximal $\mathrm{pH}$ electrode placed $5 \mathrm{~cm}$ above the lower esophageal sphincter (LES). The 6 impedance measuring segments were located 3, 5, 7, 9, 15, and $17 \mathrm{~cm}$ above the LES. The times of meals, changes in body posture and occurrence of symptoms were recorded by the patients. According to the total percentage time of esophageal $\mathrm{pH}$ values below 4, pathologic esophageal acid reflux $(\mathrm{pH}[+])$ was present if the total percentage time of esophageal $\mathrm{pH}$ below 4 exceeded 4\%. A symptom association probability (SAP) exceeding $95 \%$ was defined as positive.

The patients were categorized into the following groups: erosive esophagitis group EE group (mucosal lesion under upper endoscopy according to the Los Angeles classification); NERD group (pathologic esophageal acid exposure); hypersensitive esophagus (HO) group (SAP positive to acid reflux); and functional heartburn $(\mathrm{FH})$ group (no mucosal lesion on upper endoscopy, negative 24-hour MII-pH monitoring, and negative SAP).

\section{Salivary Collection and Pepsin Measurement}

The patients underwent 24-hour MII-pH and were asked to collect saliva before bedtime, after awakening (before eating and drinking and before brushing teeth), and at the time of symptom manifestation. For healthy volunteers who manifested no symptoms, saliva was collected 0.5 hours after meals rather than at the time of symptom manifestation. Two milliliters of saliva was collected into tubes containing $0.5 \mathrm{~mL}$ of $0.01 \mathrm{M}$ citric acid. All samples were centrifuged $4000 \mathrm{rpm}$ for 5 minutes on the same day the subjects returned the samples, together with the reflux monitoring system. The collected supernatant was stored at $-80^{\circ} \mathrm{C}$ until further analysis. Salivary pepsin concentrations were detected using the human pepsin ELISA kit (Catalog No. CSB-E08919h; CUSABIO, WuHan, HuBei, China). The analyses were performed with the researchers blinded to whether subjects were healthy volunteers or patients with GERD and to any reflux monitoring parameter.

\section{Statistical Methods}

The IS and salivary pepsin concentrations are presented as medians (quartile). SPSS version 17.0 (IBM Corp, Armonk, NY, USA) software was used to analyze the data. Kruskal-Wallis test were used to compare the parameters and Pearson's correlation was employed to compare the correlation between IS and salivary pep$\sin . P$-values $<0.05$ were considered statistically significant.

Table. Demographic Characteristics of All Subjects

\begin{tabular}{lccccc}
\hline & EE $(\mathrm{n}=10)$ & NERD $(\mathrm{n}=10)$ & HE $(\mathrm{n}=10)$ & FH $(\mathrm{n}=10)$ & $\mathrm{HV}(\mathrm{n}=10)$ \\
\hline Gender $(\mathrm{F}: \mathrm{M})$ & $5: 5$ & $4: 6$ & $4: 6$ & $6: 4$ & $8: 2$ \\
Age (mean $\pm \mathrm{SD}, \mathrm{yr})$ & $50.80 \pm 10.33$ & $45.20 \pm 14.37$ & $42.10 \pm 10.42$ & $48.30 \pm 9.33$ & $24.30 \pm 2.00$ \\
BMI (mean $\left.\pm \mathrm{SD}, \mathrm{kg} / \mathrm{m}^{2}\right)$ & $24.84 \pm 1.94$ & $22.52 \pm 2.00$ & $22.40 \pm 3.39$ & $23.21 \pm 4.02$ & $20.03 \pm 2.41$ \\
\hline
\end{tabular}

EE, Erosive esophagitis; NERD, non-erosive reflux disease; HE, hypersensitive esophagus; FH, functional heartburn; HV, healthy volunteer; F, female; M, male; BMI, body mass index. 
Results

Demographic Characteristics

A total of 45 patients were recruited into the study; 5 were excluded because of abnormal findings in the upper GI endoscopy test (1 gastric ulcer, 3 duodenal ulcer, and 1 stomach submucosal mass). Among the healthy volunteers, 1 was excluded because of an artifact during the 24-hour MII-pH monitoring. Thus, 40 patients with reflux symptoms ( 21 males; mean age: $46.60 \pm 11.33$ years; mean body mass index: $23.24 \pm 3.03 \mathrm{~kg} / \mathrm{m}^{2}$ ) and 10 healthy adults
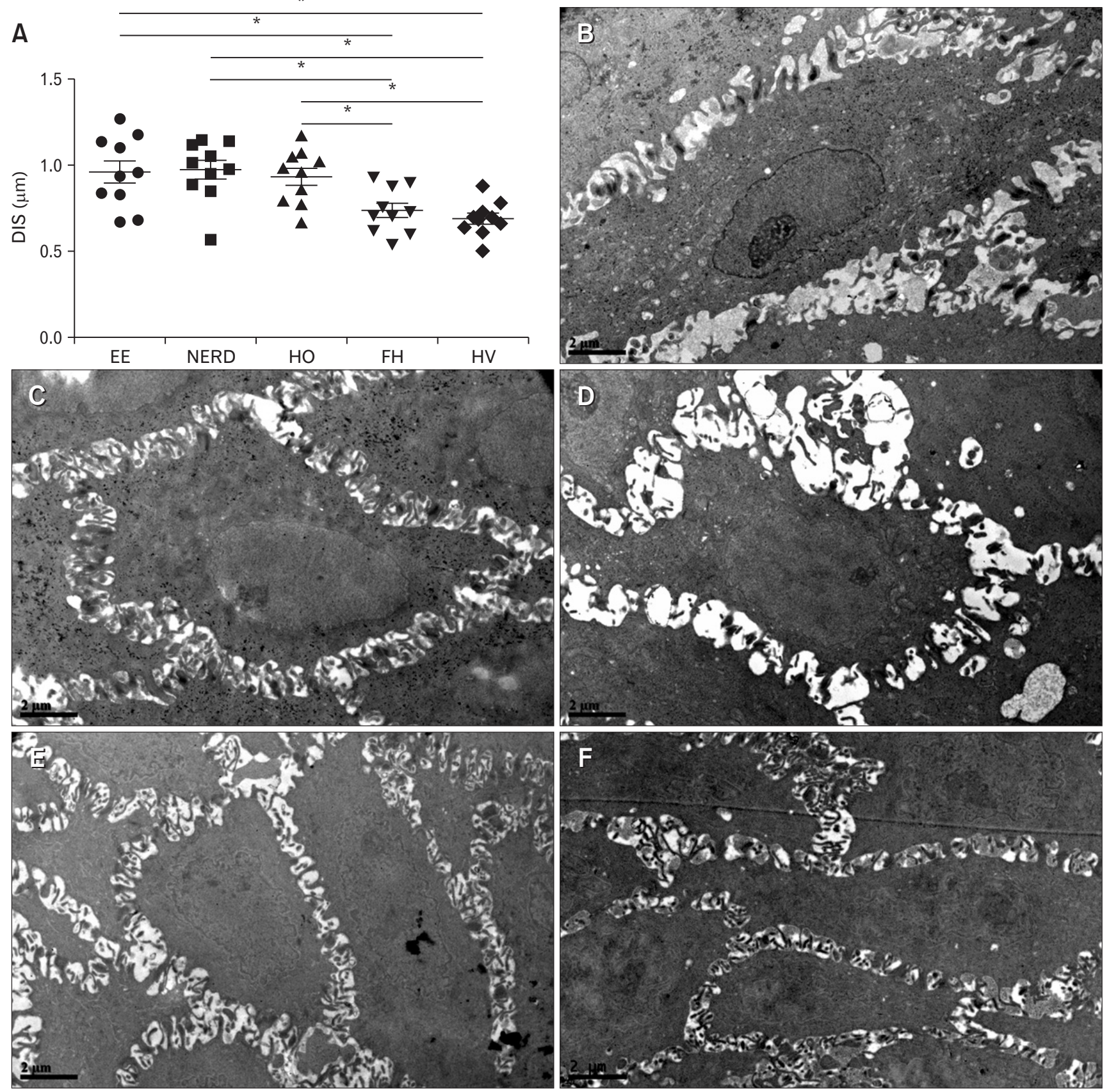

Figure 1. (A) Comparison of intercellular space among the esophagitis (EE), non-erosive reflux disease (NERD), hypersensitive esophagus (HO), functional heartburn $(\mathrm{FH})$, and healthy volunteer (HV) groups. (B-F) Electron microscope image of EE, NERD, HO, FH, and HV groups. DIS, dilated intercellular spaces. 
( 2 males; mean age: $24.30 \pm 2.00$ years; mean body mass index: $20.03 \pm 2.41 \mathrm{~kg} / \mathrm{m}^{2}$ ) were included in the final analysis. The 40 patients consisted of 10 patients with EE, 10 patients with NERD, 10 patients with $\mathrm{HO}$, and 10 patients with $\mathrm{FH}$ patients (Table).

A

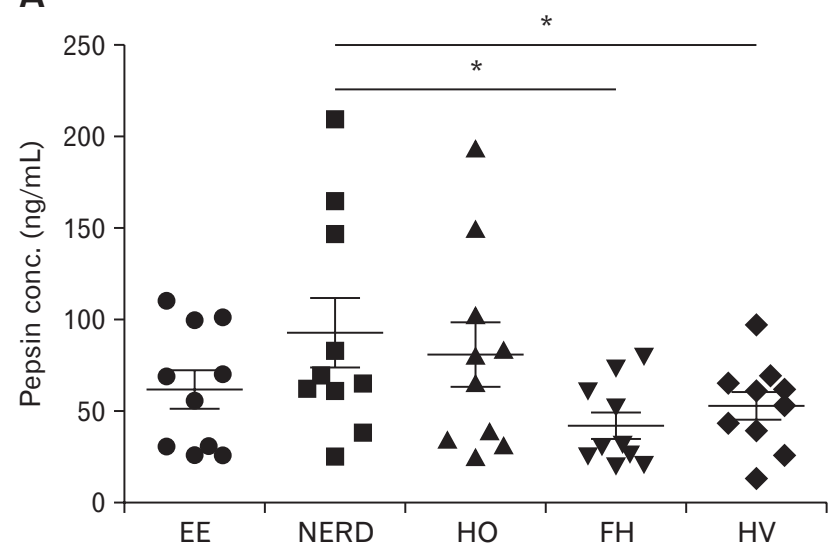

C

At the time of symptoms

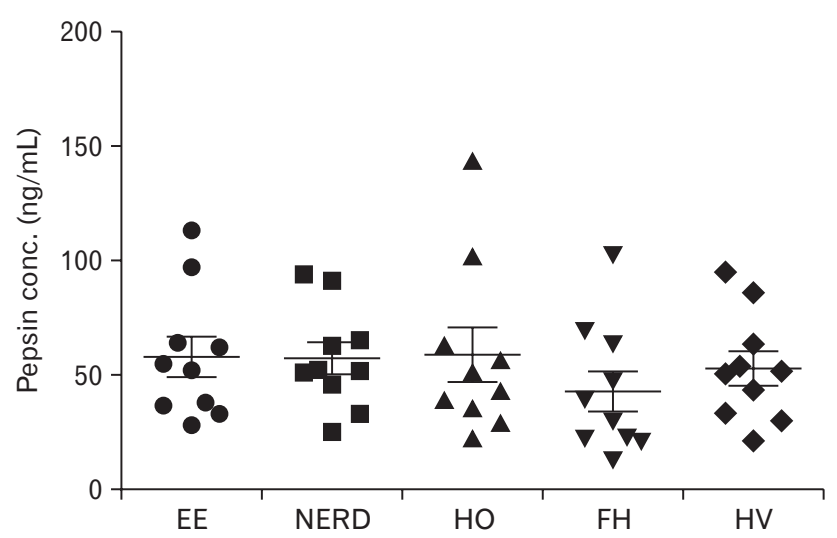

E

Average

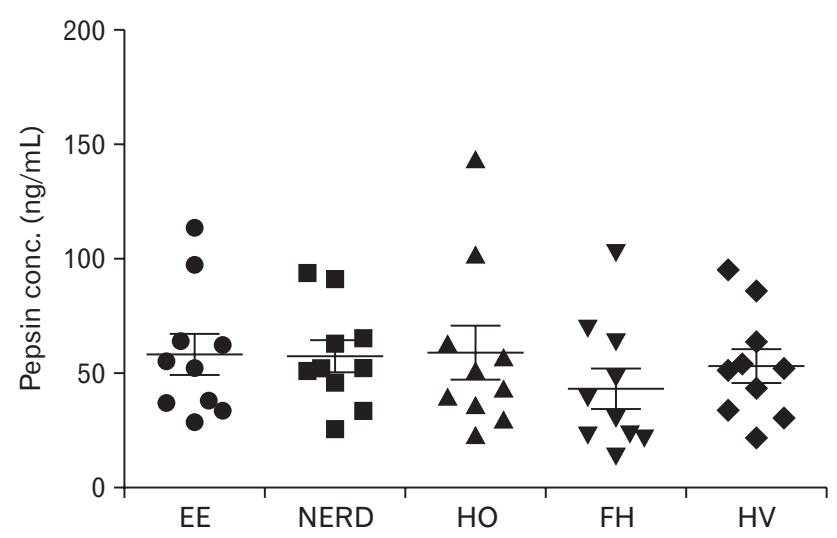

Comparison of Intercellular Space Diameter Measurements (see Fig. 1)

The IS in EE, NERD, HO, FH, and the healthy volunteer groups had the following IS measurements: 0.95 (0.79-1.15), 1.00 (0.88-1.12), 0.97 (0.78-1.05), $0.72(0.61-0.88)$, and 0.70 (0.63-
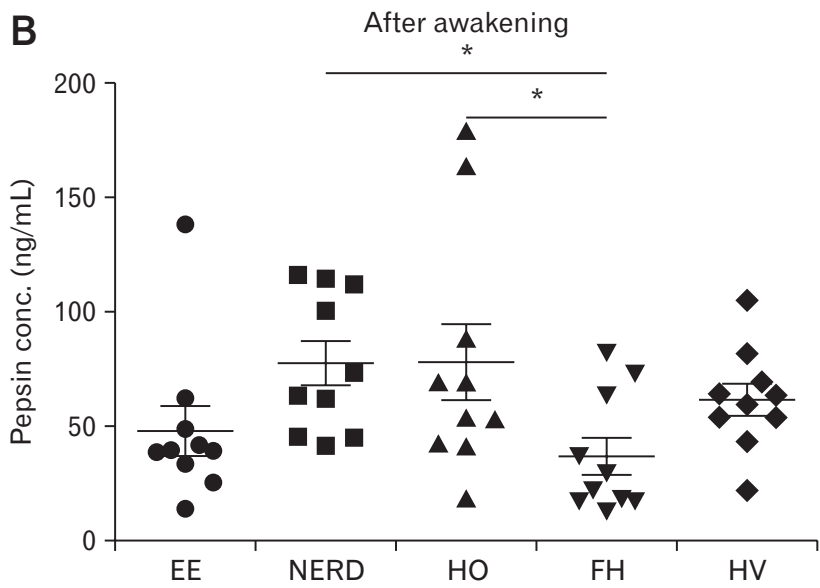

D The maximal pepsin concentration

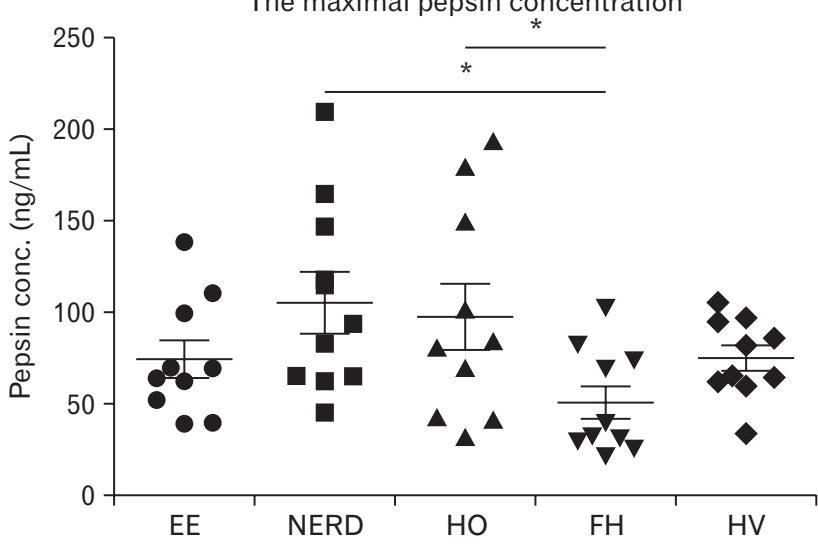

Figure 2. Salivary pepsin concentration (conc.) in different time points. EE, esophagitis; NERD, non-erosive reflux disease; HO, hypersensitive esophagus; $\mathrm{FH}$, functional heartburn; HV, healthy volunteer. ${ }^{*} P<0.05$. 
0.74) $\mu \mathrm{m}$, respectively. The measurements were greater in the EE, $\mathrm{NERD}$, and $\mathrm{HO}$ groups than in the $\mathrm{FH}$ and healthy volunteer groups, significant differences were indicated (Fig. 1).

\section{Comparison of Salivary Pepsin (see Fig. 2)}

\section{Before bedtime}

A total of 50 samples were collected, saliva pepsin concentrations measured before bedtime in the $\mathrm{EE}, \mathrm{NERD}, \mathrm{HO}, \mathrm{FH}$, and healthy volunteer groups were 62.46 (31.19-100.12), 67.95 (55.57151.39), 73.03 (34.01-114.10), 31.59 (23.93-63.98), and 57.08 (35.78-66.43) ng/mL; no significant difference $(P=0.101)$ in salivary pepsin before bedtime was indicated. However, the NERD and $\mathrm{HO}$ groups had a higher salivary pepsin concentration than the FH group ( $P=0.019$ and $P=0.035$, respectively).
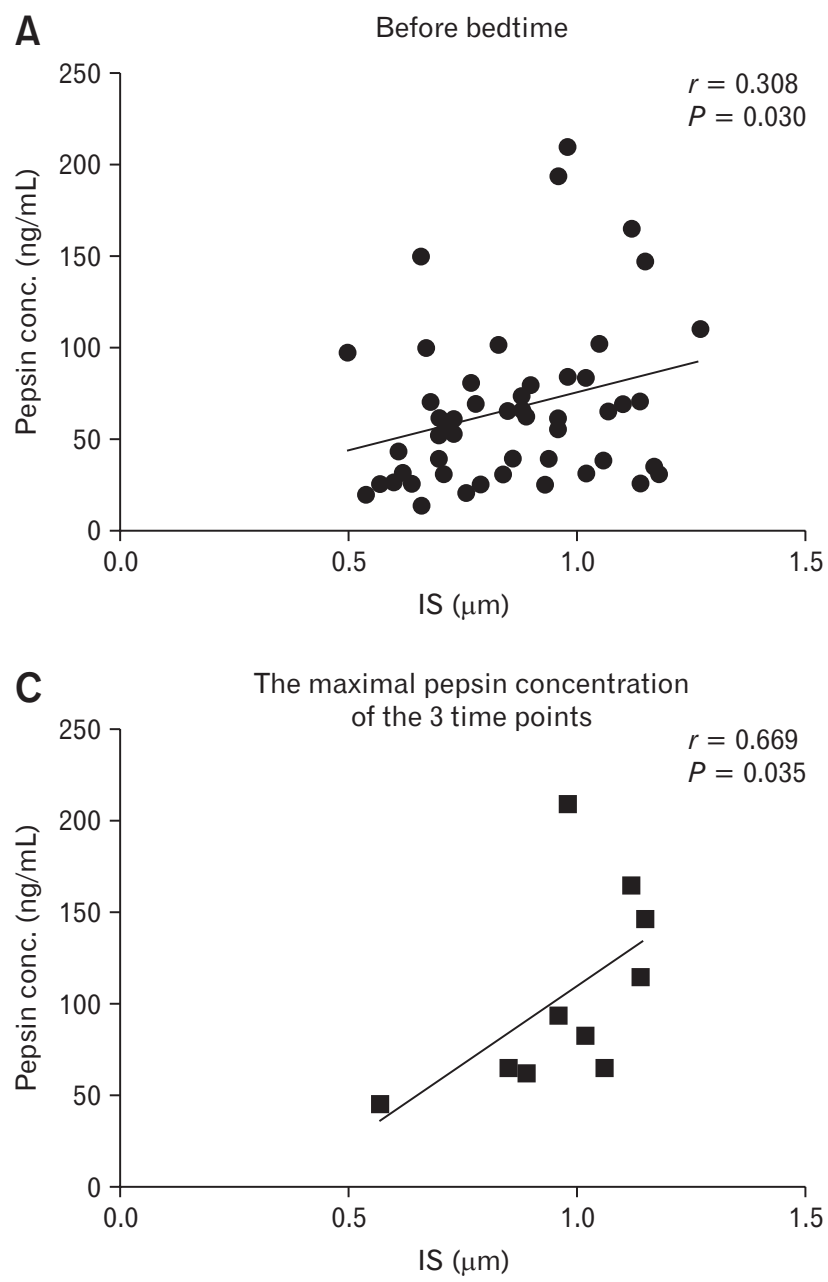

\section{After awakening}

A total of 50 samples were collected. The pepsin concentrations measured after awakening in EE, NERD, HO, FH, and healthy volunteer groups were 39.08 (25.76-52.30), 68.50 (45.29-112.89), 61.54 (42.24-107.27), 25.36 (16.77-65.59), and 61.51 (51.25$72.41) \mathrm{ng} / \mathrm{mL}$, significant difference $(P=0.011)$ in salivary pepsin after awakening was indicated. Compared with the FH group, the NERD and $\mathrm{HO}$ groups showed higher salivary pepsin concentrations ( $P=0.007$ and $P=0.035$, respectively).

\section{At the time of symptoms arise}

A total of 50 samples were collected. The salivary pepsin concentrations at the time of symptom manifestation in the EE, NERD, HE, FH, and healthy volunteer groups were 53.64 (36.06-72.31), 52.13 (42.77-71.85), 47.76 (34.26-73.10), 34.35 (22.24-64.87), and $51.46(32.73-69.06) \mathrm{ng} / \mathrm{mL}$. No significant difference in salivary pepsin at the time of symptom manifestation was

B

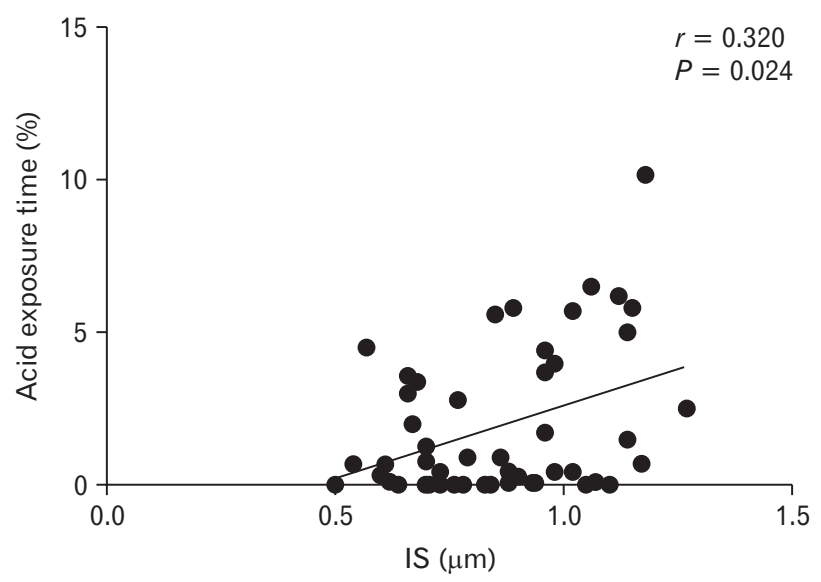

- All subjects

- NERD patients 
indicated $(P=0.593)$.

\section{The Maximal Pepsin Concentration During 24-hour Monitoring}

The maximal salivary pepsin concentration during the 24-hour monitoring of the EE, NERD, HE, $\mathrm{FH}$, and healthy volunteer groups were 66.55 (49.06-102.35), 88.47 (64.59-151.39), 82.26 (42.24-157.10), 35.60 (28.4-75.56), and 73.72 (61.53-95.57) ng/ $\mathrm{mL}$. No significant difference in the maximal salivary pepsin concentrations during 24-hour monitoring was indicated $(P=0.072)$, but compared with the FH group, the NERD, and HO groups showed higher salivary pepsin concentrations $(P=0.015$ and $P=$ 0.029 , respectively).

\section{Average Pepsin Concentration During the 24-hour Monitoring}

The average pepsin concentration during the 24-hour monitoring of patients in the EE, NERD, HE, $\mathrm{FH}$, and healthy volunteer groups were 48.89 (37.59-67.81), 71.25 (52.55-99.67), 60.76 (37.78-104.88), 27.90 (21.82-63.07), and 55.62 (50.98-65.24) ng/ $\mathrm{mL}$. No significant difference in average salivary pepsin concentrations during the 24-hour monitoring was indicated ( $P=0.056$ ), The NERD and HO groups had the higher level of average pepsin level compared with the $\mathrm{FH}$ group $(P=0.011$ and $P=0.043$, respectively).

\section{The Relationship Between Intercellular Spaces and Salivary Pepsin Concentrations}

A weak correlation exhibited between the IS and salivary pepsin concentration before bedtime of all subjects $(r=0.308, P=0.030)$. Among patients with pathologic acid exposure, IS correlated with maximal pepsin concentration during the 24-hour monitoring ( $r=$ 0.669, $P=0.035$ ) (Fig. 3). However, no correlation was found in other subgroups, the data are presented in the Supplementary Table.

A weak correlation was determined between IS and acid exposure time (AET) $(r=0.320, P=0.024)$ among all subjects. However, no correlation was obtained between AET and salivary pepsin concentration.

\section{Discussion}

Current diagnostic methods for GERD has some limitations. In the clinical setting, a simpler and less costly method that can reflect mucosal damage is required. Previous studies found that in patients with symptoms suggestive of GERD, testing for salivary pepsin may facilitate the diagnosis of GERD.${ }^{14}$ However, a high variation of salivary pepsin concentration was observed, and whether salivary pepsin could reflect mucosal damage is undermined.

Earlier studies demonstrated that under the microscope, various ultrastructural lesions with DIS were commonly observed. ${ }^{8}$ Caviglia et $\mathrm{al}^{17}$ suggested that DIS is a feature present in patients with NERD, regardless of esophageal acid exposure and can be considered as an objective, structural marker of GERD symptoms. Carney et $\mathrm{al}^{17,18}$ observed that acid was perfused in vivo into the lower esophagus, dilation of IS and reduction in potential difference were observed, which might have been caused by the inhibition of sodium transport attributed to injury from acid, bile, or pepsin. In acid-exposed tissues, dilation of IS was observed in both animal models and humans. ${ }^{19,20}$ In patients with EE and NERD, DIS was detected using transmission electron photomicrographs. ${ }^{16,20} \mathrm{In}$ the present study, we found that the EE, NERD, and HO groups had wider IS than those of the $\mathrm{FH}$ and healthy volunteer groups, significant differences were indicated. The DIS could suggest that patients with reflux disease exhibited increased paracellular permeability in the esophageal epithelium. ${ }^{21}$ Sensory neurons in the esophageal epithelium reside within the intercellular spaces; thus, an increase in paracellular permeability can potentially explain the presence of heartburn symptoms during esophageal acid exposure in patients with NERD. ${ }^{21}$ Weijenborg et $\mathrm{al}^{22}$ also demonstrated that compared with healthy volunteers, patients with GERD are more sensitive to acid. This occurrence is related to the damage to esophageal mucosal integrity. Treatment with PPIs not only heals the erosion of the esophageal mucosa, but promotes the normalization of DIS as well, thereby reducing the stimulation caused by the reflux events, and relieving the symptoms. ${ }^{23}$ Thus, DIS can be used as an objective indicator for NERD. ${ }^{11,12}$ Characteristic features in esophageal endoscopic biopsies have been proposed for a differential diagnosis between NERD and $\mathrm{FH}^{24}$ However, evaluation of the DIS is invasive and expensive, and can only be conducted by endoscopy.

Saliva pepsin has recently been proposed as a non-invasive diagnostic method for reflux disease. ${ }^{14}$ In the current study, we found that healthy volunteers had low pepsin concentrations, suggesting that physiological reflux can bring small amounts of pepsin into the oral cavity. Patients with reflux-related symptoms (NERD and $\mathrm{HO}$ ) have higher pepsin concentrations compared with the $\mathrm{FH}$ and healthy volunteer groups. Similar to previous studies, our study showed that patients with $\mathrm{FH}$ had low pepsin concentrations, this finding is closely relevant to that in clinical practice, as having all 
negative pepsin samples (less than $16 \mathrm{ng} / \mathrm{mL}$ ) suggested that the symptoms might not be due to reflux. ${ }^{14}$ These findings may lead us to avoid unnecessarily prolonged PPI treatment or anti-reflux surgery for these patients. A recent study indicated that the DIS is a characteristic GERD feature and can also be observed as reflux esophagitis progressed in patients under study. ${ }^{25}$ At the time point of symptom manifestation for patients and the time point of half an hour after dinner for health volunteers, the pepsin concentrations are similar. This effect could be attributed to the high incidence of transit LES relaxation rate in both healthy volunteer and patient groups. In addition, previous studies showed similar gastric pepsin secretion rates in healthy volunteers and patients with esophagitis. ${ }^{25}$ No findings indicate the EE group has higher pepsin concentration than the $\mathrm{FH}$ and healthy volunteer groups, the reason may be that none of the patients with esophagitis had a grade of $\mathrm{C}$ or $\mathrm{D}$ according to Los Angeles classification of esophagitis. Most patients with GERD were found to have mild esophagitis or NERD in China, particularly South China, as confirmed by our previously reported epidemiology study. ${ }^{25}$

A weak correlation was found between IS and pepsin concentrations before bedtime in all subjects $(r=0.308, P=0.030)$. In patients with pathologic acid exposure, IS correlated with the maximal pepsin level during the 24-hour monitoring $(r=0.669, P=$ 0.035 ). A recently published review by Vakil ${ }^{26}$ suggested that use of questionnaires for the diagnosis of uncomplicated GERD in primary care is economical and convenient to a patient. Saliva testing combined with specific questionnaires on GERD can keep the costs and inconvenience to patients to a minimum. ${ }^{14}$ The results obtained in the present study suggested a weak relationship between salivary pepsin concentration and DIS.

In our study, we confirmed previous findings on the lack of correlation between salivary pepsin concentration and total esophageal acid exposure. About one-third of healthy asymptomatic individuals have detectable pepsin in the saliva. ${ }^{14}$ The average pepsin concentration of the healthy volunteers in our study was also higher than $16 \mathrm{pg} / \mathrm{mL}$, which is the cut-off value for pepsin positivity. ${ }^{14}$ This result suggest that physiological reflux can bring small amounts of pepsin into the oral cavity. Similar to DIS, AET describes the consequences of reflux, whereas pepsin in the saliva describes the occurrence of reflux regardless of mucosal consequences, which can be affected by other factors, such as clearance and mucus.

This study has several limitations. The "gold standard" selected for considering "real" GERD was reflux monitoring. However, reflux monitoring cannot achieve a $100 \%$ sensitivity and specificity because of significant day-to-day variability in reflux parameters, rendering this test inaccurate for AET detection in patients. Another limitation is the lack of follow-up data. In addition, we failed to recruit age-matched healthy volunteers. The healthy volunteers were in the young age bracket, which could be regarded as a factor affecting the reliability of the results. However, we conducted a subgroup analysis among patients in the 19-40 years age range, and patients older than 40 years, and no significant differences in acid exposure, salivary pepsin concentration, and IS were indicated between these 2 subgroups. Moreover, the patients in the younger age bracket had significant acid exposure and IS compared with healthy volunteers. Regardless, we acknowledge that this is still a potential confounding variable.

To conclude, a higher salivary pepsin concentration was found in patients with NERD than in patients with $\mathrm{FH}$, and salivary pepsin concentrations correlated with severity of mucosal integrity impairment. The findings suggest that the higher the pepsin concentration, the more likely the severity of mucosal damage. In addition, low salivary pepsin concentrations could help identify patients with $\mathrm{FH}$. However, further research is needed to elucidate the factors that determine salivary pepsin concentration.

\section{Supplementary Material}

Note: To access the supplementary table mentioned in this article, visit the online version of Journal of Neurogastroenterology and Motility at http://www.jnmjournal.org/, and at https://doi. org/10.5056/jnm16178.

Financial support: This study was supported by China National Natural Science Foundation 81400582 (Yinglian Xiao) and the Research Fund for the Doctoral Program of Higher Education of China 20110171120080 (Yinglian Xiao).

\section{Conflicts of interest: None.}

Author contributions: Acquisition of data, analysis, and drafting: Yuwen Li; acquisition of data and analysis: Chenxi Xie; study concept, editing the English language, and finalizing the manuscript: Daniel Sifrim; and acquisition of data, analysis, drafting, study supervision, and finalizing the manuscript: Minhu Chen and Yinglian Xiao.

\section{References}

1. Vakil N, van Zanten SV, Kahrilas P, Dent J, Jones R. The Montreal 
definition and classification of gastroesophageal reflux disease: a global evidence-based consensus. Am J Gastroenterol 2006;101:1900-1920.

2. Dent J, El-Serag H, Wallander MA, Johansson S. Epidemiology of gastro-oesophageal reflux disease: a systematic review. Gut 2005;54:710717.

3. Johansson KE, Ask P, Boeryd B, Fransson S-G, Tibbling L. Oesophagitis, signs of reflux, and gastric acid secretion in patients with symptoms of gastro-oesophageal reflux disease. Scand J Gastroenterol 1986;21:837847.

4. Kahrilas PJ, Quigley EM. Clinical esophageal $\mathrm{pH}$ recording: a technical review for practice guideline development. Gastroenterology 1996;110:1982-1996.

5. Lawenko RM, Lee YY. Evaluation of gastroesophageal reflux disease using the Bravo capsule $\mathrm{pH}$ system. J Neurogastroenterol Motil 2016;22:25-30.

6. Knight J, Lively MO, Johnston N, Dettmar PW, Koufman JA. Sensitive pepsin immunoassay for detection of laryngopharyngeal reflux. Laryngoscope 2005;115:1473-1478.

7. Wong WM, Fass R. Extraesophageal and atypical manifestations of GERD. J Gastroenterol Hepatol 2004;19(suppl 3):S33-S43.

8. Yerian L, Fiocca R, Mastracci L, et al. Refinement and reproducibility of histologic criteria for the assessment of microscopic lesions in patients with gastroesophageal reflux disease: the Esohisto Project. Dig Dis Sci 2011;56:2656-2665.

9. Van Malenstein H, Farré R, Sifrim D. Esophageal dilated intercellular spaces (DIS) and nonerosive reflux disease. Am J Gastroenterol 2008;103:1021-1028.

10. Knowles $\mathrm{CH}$, Aziz Q. Visceral hypersensitivity in non-erosive reflux disease. Gut 2008;57:674-683.

11. Calabrese C, Bortolotti M, Fabbri A, et al. Reversibility of GERD ultrastructural alterations and relief of symptoms after omeprazole treatment. Am J Gastroenterol 2005;100:537-542.

12. Kahrilas PJ. Dilated intercellular spaces: extending the reach of the endoscope. Am J Gastroenterol 2005;100:549-550.

13. Samuels TL, Johnston N. Pepsin as a causal agent of inflammation during nonacidic reflux. Otolaryngolo Head Neck Surg 2009;141:559-563.
14. Hayat JO, Gabieta-Somnez S, Yazaki E, et al. Pepsin in saliva for the diagnosis of gastro-oesophageal reflux disease. Gut 2015;64:373-380.

15. Streckfus CF, Bigler LR. Saliva as a diagnostic fluid. Oral Dis 2002;8:6976.

16. Tobey NA, Carson JL, Alkiek RA, Orlando RC. Dilated intercellular spaces: a morphological feature of acid reflux--damaged human esophageal epithelium. Gastroenterology 1996;111:1200-1205.

17. Caviglia R, Ribolsi M, Maggiano N, et al. Dilated intercellular spaces of esophageal epithelium in nonerosive reflux disease patients with physiological esophageal acid exposure. Am J Gastroenterol 2005;100:543548.

18. Carney CN, Orlando RC, Powell DW, Dotson MM. Morphologic alterations in early acid-induced epithelial injury of the rabbit esophagus. Lab Invest 1981;45:198-208.

19. Ferreira KT, Hill BS. The effect of low external $\mathrm{pH}$ on properties of the paracellular pathway and junctional structure in isolated frog skin. J Physiol 1982;332:59-67.

20. Hopwood D, Milne G, Logan KR. Electron microscopic changes in human oesophageal epithelium in oesophagitis. J Pathol 1979;129:161-167.

21. Orlando RC. Pathophysiology of gastroesophageal reflux disease. J Clin Gastroenterol 2008;42:584-588.

22. Weijenborg PW, Smout AJ, Verseijden C, et al. Hypersensitivity to acid is associated with impaired esophageal mucosal integrity in patients with gastroesophageal reflux disease with and without esophagitis. Am J Gastrointest Liver Physiol 2014;307:G323-G329.

23. Xue Y, Zhou L-Y, Lin S-R. Dilated intercellular spaces in gastroesophageal reflux disease patients and the changes of intercellular spaces after omeprazole treatment. Chin Med J 2008;121:1297-1301.

24. Savarino E, Zentilin P, Mastracci L. Light microscopy is useful to better define NERD and functional heratburn. Gut 2014;63:368.

25. Fiorucci S, Distrutti E, Di Matteo F, et al. Circadian variations in gastric acid and pepsin secretion and intragastric bile acid in patients with reflux esophagitis and in healthy controls. Am J Gastroenterol 1995;90:270276.

26. Vakil N. The initial diagnosis of GERD. Best Pract Res Clin Gastroenterol 2013;27:365-371. 